\title{
Soldadura laser de sub-conjuntos para estampagem (Tailored blanks)
}

\author{
P. Peças ${ }^{(*)}$, H. Gouveia ${ }^{(*)}$, L. Quintino ${ }^{(* *)}$, M. Rasmussen $^{(* * *)}$ y F.O. Olsen ${ }^{(* *)}$
}

Resumo: A soldadura laser assume um papel cada vez mais importante na indústria automóvel, principalmente para a fabricação de sub-conjuntos constituídos por várias partes de chapa de diferentes espessuras (e diferentes materiais), que depois de estampados constituem um componente para integrar num veículo. Descreve-se neste artigo o trabalho de investigação de enformabilidade de chapa de aço de baixo carbono soldada por laser de $\mathrm{CO}_{2}$, nas espessuras de 1,25 e $0,75 \mathrm{~mm}$. Apresenta-se uma descrição do comportamento das chapas soldadas por laser em diferentes testes de enformação, e a influência dos defeitos das soldaduras (desalinhamento e queda do banho-undercut) no comportamento à enformação. A qualidade é avaliada pela medição da extensão limite e da extensão limite efectiva no material base e no material soldado, que serão representadas num diagrama de limite de enformabilidade.

Palavras-chave: Soldadura laser. Tailored blanks. Chapa fina.

\section{Laser welding of tailored blanks}

\begin{abstract}
Laser welding has an incrising role in the automotive industry, namely on the sub-assemblies manufacturing. Several sheet-shape parts are laser welded, on a dissmilar combination of thicknesses and materials, and are afterwads formed (stamped) being transformed in a vehicle body component. In this paper low carbon $\mathrm{CO}_{2}$ laser welding, on the thicknesses of 1,25 and $0,75 \mathrm{~mm}$, formability investigation is described. There will be a description of how the laser welded blanks behave in different forming tests, and the inlfuence of misalignment and undercut on the formibility. The quality is evaluated by measuring the limit strain and limit effective strain for the laser welded sheets and the base material, which will be presented in a forming limit diagram.
\end{abstract}

Keywords: Laser Welding. Tailored blanks. Thin thickness sheets.

\section{INTRODUÇÃO}

A soldadura laser é um processo competitivo na união de chapa galvanizada de pequena espessura. As velocidades atingidas e a baixa entrega térmica ao material base são as principais vantagens do processo. No caso particular da soldadura por laser de $\mathrm{CO}_{2}$, utilizado na execução experimental deste trabalho, a grande gama de equipamentos com diversas potências disponíveis, torna esta tecnologia bastante flexível. O aumento de utililização da soldadura laser promove o incremento da produtividade, precisão e qualidade das operações de soldadura.

A tecnologia laser tem evoluído de forma positiva em todo o mundo, visto que cobre várias aplicações, tais como o corte, a marcação, os

(*) Instituto de Soldadura e Qualidade (Portugal)

(**) Instituto Superior Técnico-Secção de Tecnologia Mecânica (Portugal)

(***) The Technical University of Denmark (Dinamarca) tratamentos superficiais, a furação e obviamente a soldadura. De entre os três tipos de laser mais utilizados em processamento de materiais $\left(\mathrm{CO}_{2}\right.$, $\mathrm{Nd}$ :YAG e excímeros), o laser de $\mathrm{CO}_{2}$ é o mais versátil, já que permite todo o tipo de operações e está disponível numa larga gama de potências (desde a dezena de Watts até $40 \mathrm{~kW}$ ). As figuras seguintes ilustram a distribuição dos equipamentos laser e das aplicações.

A soldadura por laser de $\mathrm{CO}_{2}$, oferece possibilidades únicas ao nível das velocidades de processamento, de qualidade, da geometria da soldadura, e de uniões dissimilares (em materiais e espessuras). A combinação de novos conceitos de projecto com as vantagens da soldadura laser tem elevado potencial para aplicação industrial, e em particular na indústria automóvel.

Esta indústria tem sido aquela que absorve anualmente mais equipamentos laser para operações de soldadura, pelo que é uma indústria de referência para a soldadura laser. 

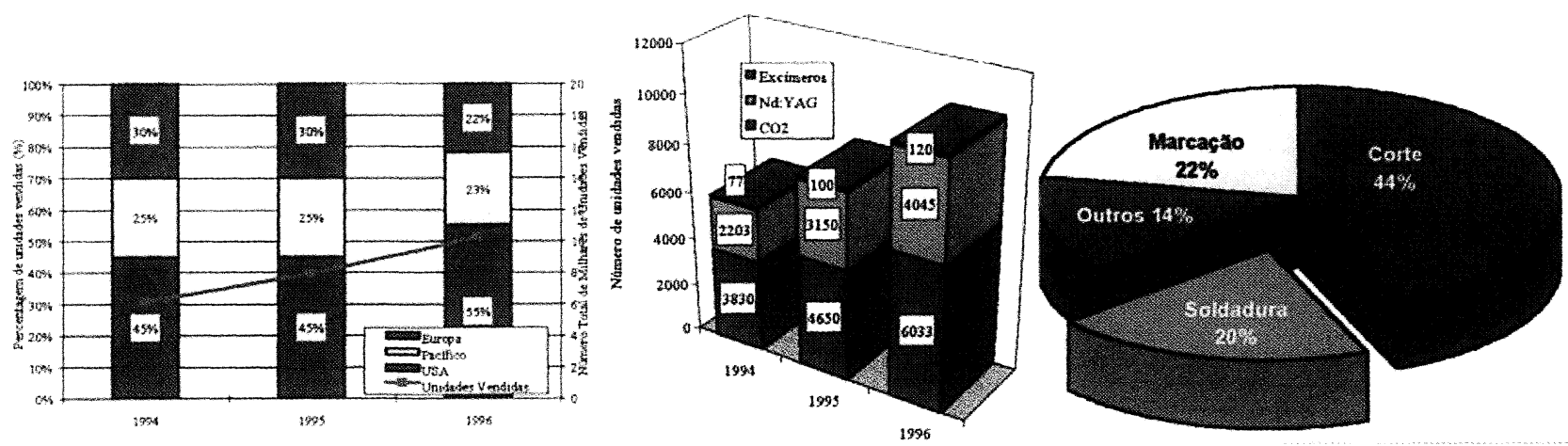

FIG. 1.-Dados de unidades vendidas e campo de aplicação dos lasers no processamento de materiais. $(1)(2)(3)(4)(5)$.

\section{FIG. 1.-Laser units market and applications in materials processing.}

A soldadura robotizada na indústria automóvel começou a ser efectuada através da soldadura por pontos, utilizando o processo de soldadura por resistência. Com o desenvolvimento da tecnologia de soldadura laser, houve vários construtores que integraram sistemas laser na soldadura por pontos. No entanto, neste tipo de união não se tira partido de toda a potencialidade da soldadura laser, já que uma das suas grandes vantagens é a velocidade de processamento.

Durante os anos 60, houve várias tentativas de integração de robots com sistemas laser, por forma a unir os componentes através cordões em vez de pontos (6). Aqui a soldadura laser tinha várias vantagens sobre a soldadura por resistência, já que os roletes (para a soldadura por resistência) não têm a mesma flexibilidade que a cabeça laser. Apesar das vantagens aparentes da soldadura laser para execução de soldadura a 3-D, os sistemas instalados deram vários problemas, devido aos exigentes requisitos de programação e de precisão (7).

Esta preocupação da indústria automóvel aparece integrada num esforço desta indústria ao nível do desenvolvimento do design das estruturas,

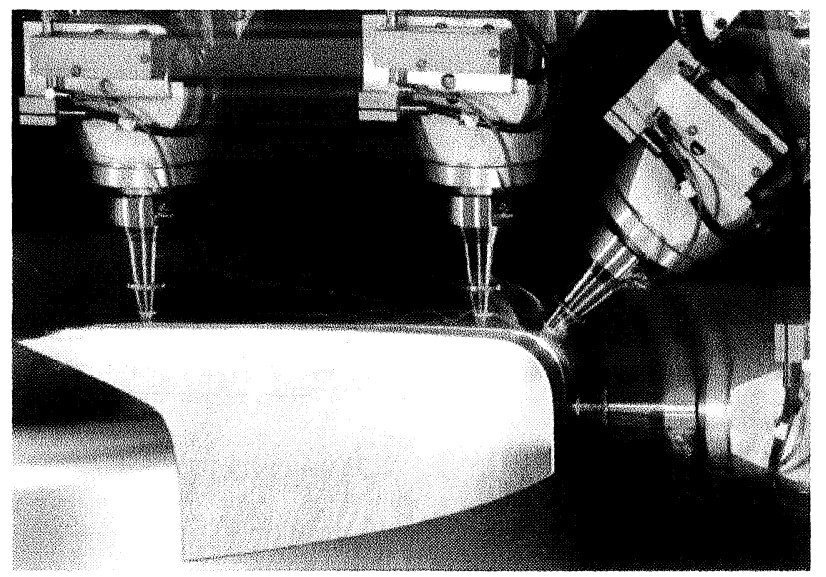

bem como da preocupação crescente com os aspectos da qualidade e da redução do peso do veículo e dos custos de produção. No fim dos anos 70 as principais tendências de desenvolvimento ao nível do design das estruturas automóveis eram:

- Melhoramentos ao nível da resistência à corrosão, através da substituição dos materiais usualmente utilizados por materiais menos propensos à corrosão como o alumínio ou aços galvanizados.

- Redução do peso, através do recurso a materiais mais leves (alumínio) na sua construção ou de um controlo mais eficaz da espessura das chapas a utilizar nas diferentes partes da estrutura.

- Melhoramentos ao nível da robustez por meio de substituição de soldadura por pontos por soldadura de cordão.

- Redução dos custos, através de uma redução do número de partes constituintes da estrutura.

Com base nestas tendências surgiu uma nova filosofia no fabrico de painéis de carroçaria - o método das tailored-blanks. Este método envolve 3 operações:

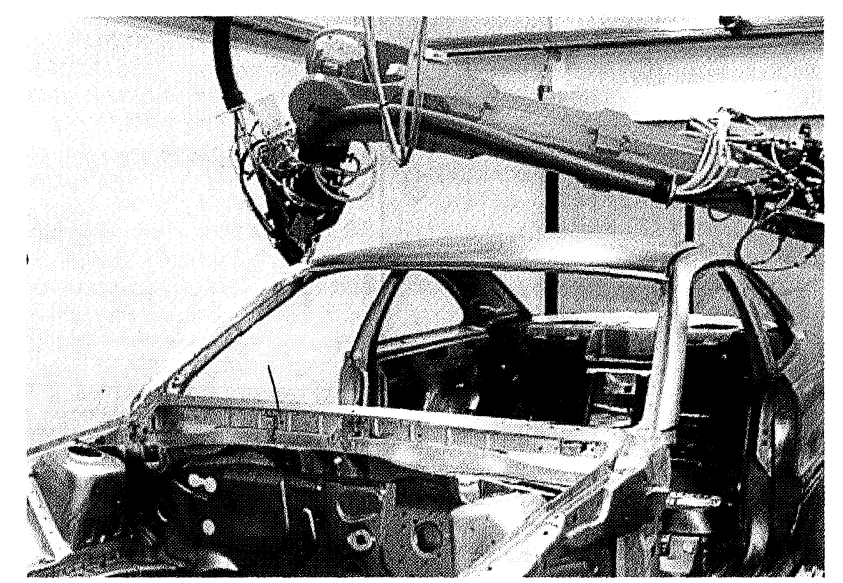

FIG. 2.- Soldadura por laser a 3-D assistida por robot, do Volvo C70 (8).

FIG. 2.- Robot aassited 3-D laser, Volvo C70. 
1. Corte de várias peças (blanks) de material/espessura/revestimento dimilares ou diferentes.

2. União das várias partes por forma a obter uma peça a 2-D composta (tailor).

3. Estampagem por forma a obter um componente complexo a 3-D.

Embora o método das tailored-blanks também se aplique ao alumínio e suas ligas, os componentes em aço galvanizado (ou compostos de aço galvanizado e aço sem revestimento) apresentam várias vantagens ao nível do custo (7), da resistência/tenacidade (mais $10 \%$ de tenacidade) e melhor soldabilidade.

Embora fosse nos anos 60 que esta tecnologia se começou a desenvolver, prinicipalmente nos E.U.A. (9), foi nos anos 80 que começou a ser utilizada em maior escala. O grupo Audi-VW foi o primeiro no Europa em 83 a instalar um sistema deste tipo, baseado na soldadura por resistência (6). Na fig. 5 estão indicados os locais na Europa onde existem sistemas instalados, sendo no total 37 sistemas.

A soldadura laser constitui um processo de soldadura muito atractivo, na medida em que permite uniões topo a topo sem sucalcos na mudança de chapa, produzindo cordões de elevada qualidade que quase não necessitam de tratamentos e/ou acabamento final. Este processo de soldadura atinge velocidades bastante elevadas em chapa fina, com a possibilidade de unir materiais dissimilares (aço revestido com aço não revestido) com espessuras dissimilares.

Dos 37 sistemas instalados na Europa, apenas 13 são baseados em soldadura laser, sendo os restantes 24 baseados na soldadura por resistência. As desvantagens da soldadura por resistência (necessidade de junta sobreposta e menor velocidade de processamento (11)), e a constante investigação do comportamento da soldadura laser tem originado uma evolução positiva na utilização desta tecnologia. A presente investigação aborda o comportamento da soldadura laser à enformação, já

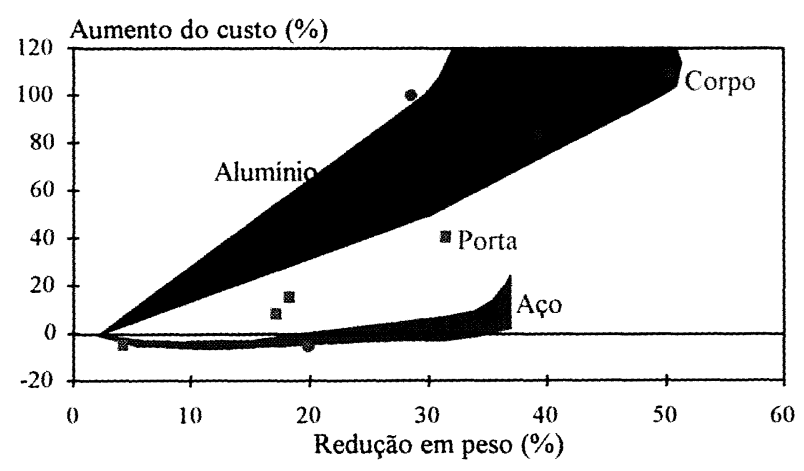

FIG. 4.- Efeito da redução do peso no custo da estrutura dos automóveis (7).

FIG. 4.- Relationship between weigth reduction and automotive body cost.

que a aplicação da tecnologia depende de um bom desempenho nesta fase de processamento.

\section{PARAMETROS DE CORTE E SOLDADURA LASER}

Quando as soldaduras laser são estampadas, é importante que o conjunto produzido possua níveis correctos de resistência, por forma a não ocorreram fracturas durante a enformação. Com o objectivo de realizar amostras para os testes de enformabilidade, os processos de corte e soldadura foram ambos optimizados. O processo de preparação de junta seleccionado foi o corte laser, uma vez que permite superfícies de corte aceitáveis para soldadura, sendo mais económico que o processo de maquinagem.

As soldadura foram avaliadas utilizando a norma DIN 8563 part II, tendo sido seleccionadas as soldaduras que apresentavam melhor qualidade. Por forma a se obter superfícies de corte com elevada qualidade foi utilizado azoto como gás de corte e baixa potência laser, evitando-se eventuais empenos originados na operação de corte. Na tabela 1 estão ilustrados os parâmetros utilizados.

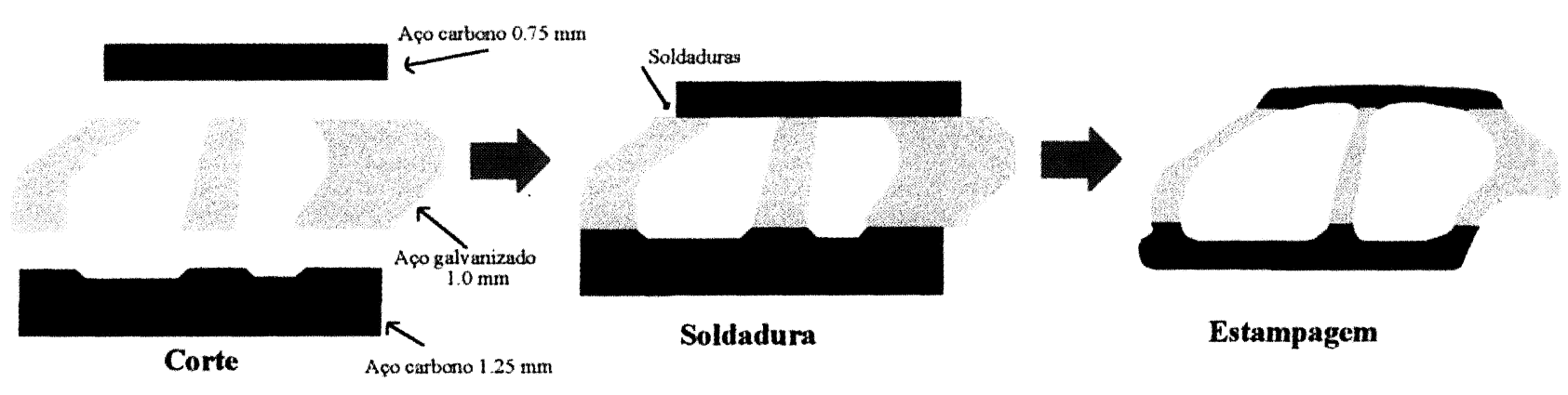

FIG. 3.-Processo de obtenção de componentes compostos complexos a 3-D pelo método das tailored-blanks. FIG. 3.-3D complex components manufacturing process based on. 


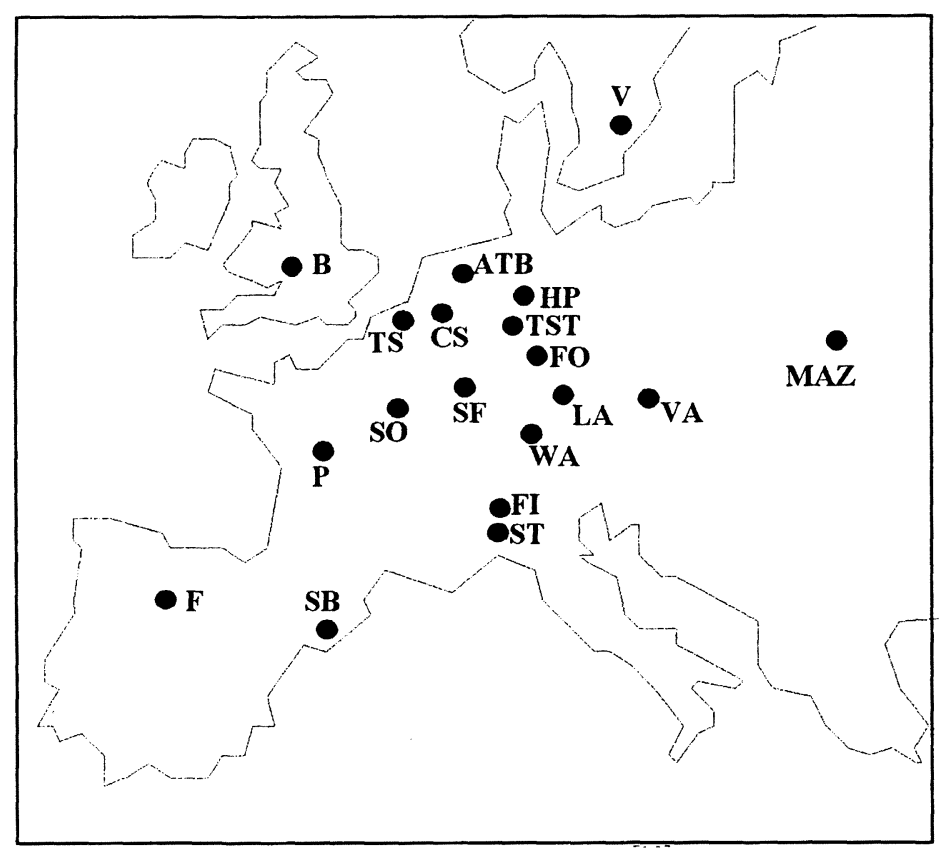

\begin{tabular}{|ll|}
\hline ATB & $=$ Automative Tailored Blanks \\
B & $=$ British Steel \\
CS & $=$ Cockerill Sambre \\
F & $=$ Fasa Renault \\
FI & $=$ Fiat Auto \\
FO & $=$ Ford \\
HP & $=$ Hoesch Platinen \\
LA & $=$ Lapple \\
MAZ & $=$ Minsk Automative Works \\
P & $=$ PSA \\
SB & $=$ Solblank \\
SF & $=$ Sofedit \\
SO & $=$ Somenor \\
ST & $=$ Grupo Stola \\
TS & $=$ Tailor Steel \\
TST & $=$ Thyssen Stahl \\
V & $=$ Volvo \\
VA & $=$ Voest Alpine Stahl \\
WA & $=$ Wackenhut \\
\hline
\end{tabular}

FIG. 5.- Sistemas de tailored-blanks na Europa (10).

FIG. 5.-Tailored-blanks systems in Europe.

TABELA I.- Parâmetros de corte e soldadura laser utilizados.

TABLE I.- Laser cutting and welding parameters.

\begin{tabular}{|l|l|l|l|l|}
\hline & \multicolumn{2}{|c|}{ CORTE } & \multicolumn{2}{c|}{ SOLDADURA } \\
\hline Espessura & $0,75 \mathrm{~mm}$ & $1,25 \mathrm{~mm}$ & $0,75 \mathrm{~mm}$ & $1,25 \mathrm{~mm}$ \\
Material & St 1203 & St 1203 & St 1203 & St 1203 \\
Potência Laser $\left(\mathrm{CO}_{2}\right)$ & $650 \mathrm{~W}$ & $575 \mathrm{~W}$ & $1500 \mathrm{~W}$ & $1500 \mathrm{~W}$ \\
Velocidade & $1100 \mathrm{~mm} / \mathrm{min}$ & $1100 \mathrm{~mm} / \mathrm{min}$ & $3000 \mathrm{~mm} / \mathrm{min}$ & $3000 \mathrm{~mm} / \mathrm{min}$ \\
Tipo de gás & Azoto & Azoto & Azoto & Azoto \\
Pressão/Caudal & 18 bar & 18 bar & $111 / \mathrm{min}$ & 11 1/min \\
\hline
\end{tabular}

\section{INFLUENCIA DO DESALINHAMENTO VERTICAL NA ENFORMABILIDADE}

Por forma a investigar como o desalinhamento vertical, resultante da soldadura laser, influência a enformabilidade, foram realizadas soldaduras em chapa de 0,75 mm de espessura do aço carbono St 1203 para posterior enformação. Por forma a obter vários níveis de desalinhamento, foi concebido o arranjo experimental ilustrado na fig. 6 .

Impuseram-se três desalinhamentos verticais: 0,3 ; 0,4 e $0,5 \mathrm{~mm}$. Em primeiro lugar realizaram-se ensaios de tracção e seguidamente testes de embutição profunda: LDH - Limit Drawing Heigth. Para os ensaios de tracção utilizaram-se provetes normalizado $\left(0,75 \times 20 \times 200 \mathrm{~mm}^{3}\right)$ retirados na direcção transversal à soldadura. Os resultados estão ilustrados na fig.7, onde se constata que um desalinhamento de $0,4 \mathrm{~mm}$ é o limite para se obterem soldaduras satisfatórias.

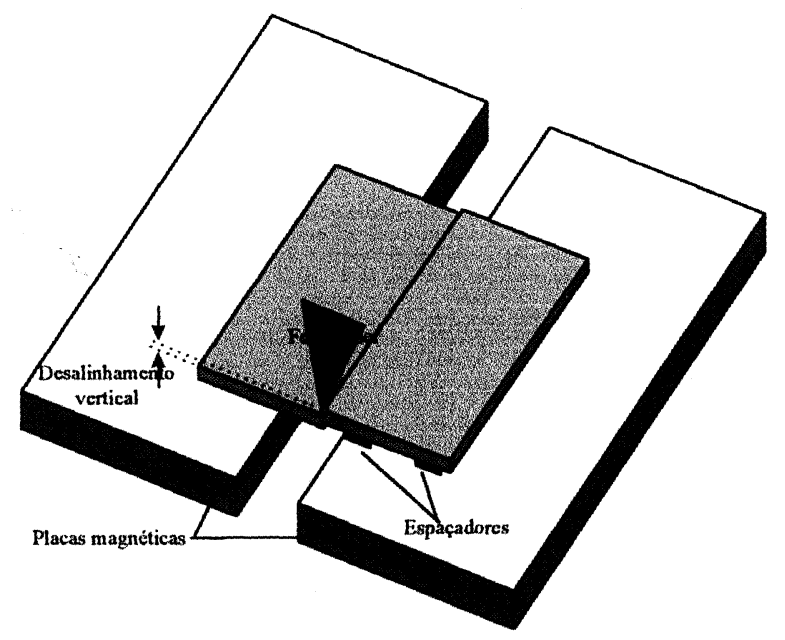

FIG. 6.- Arranjo experimental por forma a induzir desalinhamentos verticais.

FIG. 6.- Experimental set-up to produce vertical misaligned sheets. 


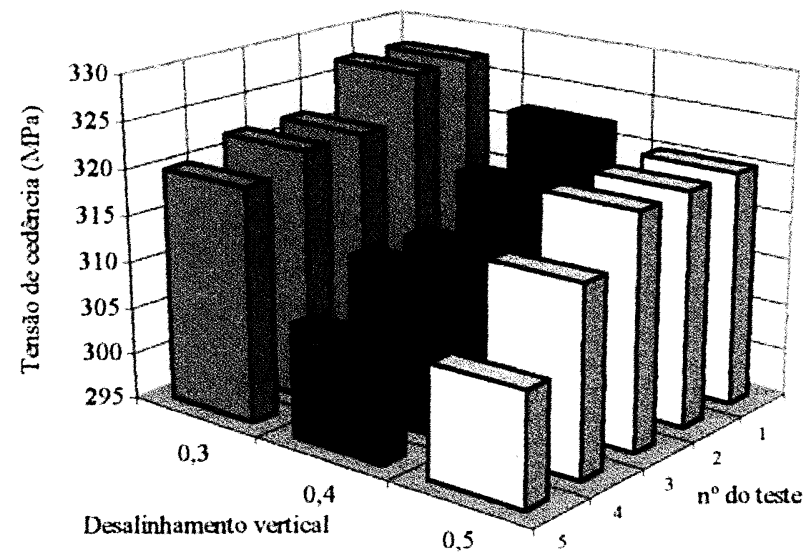

Fig. 7.- Resultados dos testes de tracção nas soldaduras com vários desalinhamentos.

FIG. 7.- Selected results from tensile tests of welds with different misalignments.

Para o teste $\mathrm{LDH}$, as chapas soldadas foram cortadas em provetes circulares com um diâmetro de cerca de $100 \mathrm{~mm}$. Os resultados estão ilustrados na fig.8, que apontam para o mesmo limite de desalinhamento que os resultados do ensaio de tracção. A conclusão é de que o valor crítico de desalinhamento é de $0,4 \mathrm{~mm}$ para aço carbono de $0,75 \mathrm{~mm}$ de espessura.

\section{INFLUENCIA DA QUEDA DO BANHO NA ENFORMABILIDADE}

Por forma a investigar como a queda do banho influência a enformabilidade, foram realizadas várias soldaduras no aço St 1203 com 0,75 de espessura, impondo vários graus de queda de banho. Aatravés da variação do caudal de gás coaxial obteram-se diversos níveis de queda de banho. O nível de queda de banho foi medido através de um rugosímetro, obtendo-se valores de queda de banho entre 10 e $74 \mathrm{~mm}$, este último obtido com um caudal de $44 \mathrm{l} / \mathrm{min}$. Acima deste

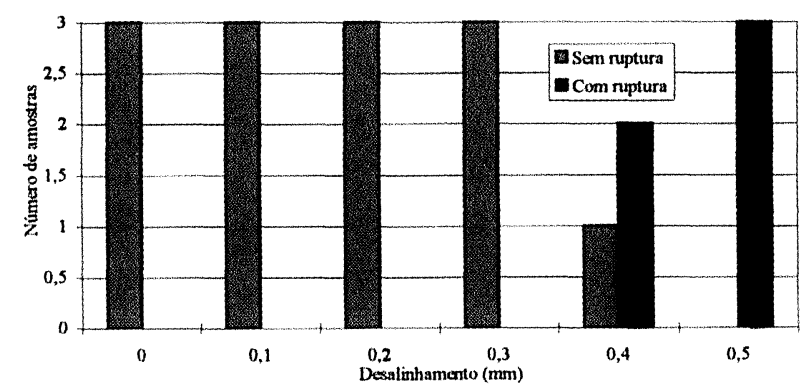

FIG. 8.- Resultados dos ensaios de LDH para diferentes desalinhamentos.

FIG. 8.- Example of a forming limit curve. valor de caudal ocorreu a furação da chapa, pelo que este é o valor de queda de banho limite. Foram realizados ensaios de tracção, seguindo-se o procedimento utilizado nos ensaios de investigação do desalinhamento vertical. Os provetes traccionados foram analisados por forma a identificar o local de ruptura, tendo-se verificado que em todos os cordões a fractura ocorreu no material base e não a partir da junta soldada. Conclui-se assim que a queda de banho é um factor que não influencia a enformabilidade.

\section{TESTES DE ENFORMABILIDADE}

Numa operação de estampagem industrial, os níveis de deformação atingidos variam em toda a extensão da chapa. Por não se tratar de um caso de deformação uniforme, o tipo de deformação varia ao longo do corpo estampado, a enformação do componente deve ser analisada com recurso à curva limite de enformabilidade (fig.9). Esta curva des'creve os limites das extensões, para as várias situações possíveis.

É possível calcular o nível das extensões num dado ponto de uma peça estampada, recorrendo a software específico de elementos finitos. Com base nas propriedades do material, estes programas permitem determinar curvas limite deenformabilidade para os materiais base, não possibilitando a análise para o caso de existir uma soldadura laser a unir as chapas. A vantagem de se utilizarem curvas limite de enformabilidade específicas para um dado componente, permite baixar os coeficientes de segurança, e consequentemente, reduzir o custo da matériaprima e o peso do veículo.

Por forma a se determinar as curvas limite de enformabilidade para o caso específico das amostras realizadas nesta investigação, foi gravado nas chapas foram uma rede de malha quadrada. As

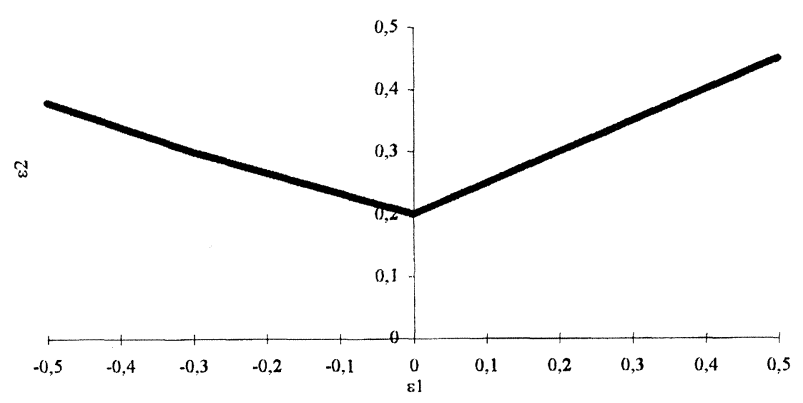

FIg. 9.- Exemplo de uma curva limite de enformabilidade.

FIG. 9.- Exemplo de uma curva limite de enformabilidade 
dimensões da rede foram medidas antes e após os testes de enformação, sendo as extensões calculadas pela equação 1 (fórmula de interpolação de Veermans (12)):

$$
\begin{aligned}
& \varepsilon=\frac{\ln 1_{1}}{1_{0}} \\
& \varepsilon^{*}=\frac{3}{4}\left(\varepsilon_{\mathrm{v} 1}+\varepsilon_{\mathrm{v} 2}\right)-\frac{3}{10}\left(\varepsilon_{\mathrm{u} 1}+\varepsilon_{\mathrm{u} 2}\right)-\frac{1}{20}\left(\varepsilon_{\mathrm{t} 1}+\varepsilon_{\mathrm{t} 2}\right)
\end{aligned}
$$

onde $l_{0}$ é a largura da rede inicial, $l_{1}$ a largura da rede após deformação, e e* é a extensão na zona instável.

Não é possível medir a extensão na zona instável (onde ocorre a fractura), pelo que é necessário estimar através de medições efectuadas na vizinhança desta zona.Esta estimativa é baseada na eq.1,e a fig.10 ilustra o local onde é medida.

Foram realizadas curvas limite de enformabilidade para ambas as espessuras seleccionadas nesta investigação. Por forma a determinar o lado esquerdo da curva $\left(\mathrm{e}_{1}\right.$ negativos $\mathrm{e}$ $\mathrm{e}_{2}$ positivos), foi utilização o ensaio de tracção, para diferentes quocientes entre extensões $\left(S=\varepsilon_{1} / \varepsilon_{2}\right)$. Estes quocientes foram impostos utilizando provetes com diferentes raios $\mathrm{R}$, entre 0,5 e Á, como se ilustra na fig. 11 . O provete com raio infinito tinha como dimensões 20 × $200 \mathrm{~mm}$. Os resultados obtidos dos testes efectuados com diferentes quocientes entre as extensões estão ilustrados na fig. 12. Para cada raio $\mathrm{R}$ foram realizados três ensaios, sendo cada ponto da curva o valor médio dos três.

Por forma a determinar o lado direito da curva limite de enformabilidade, utilizou-se o teste de embutição (bulge test), recorrendo a três matrizes

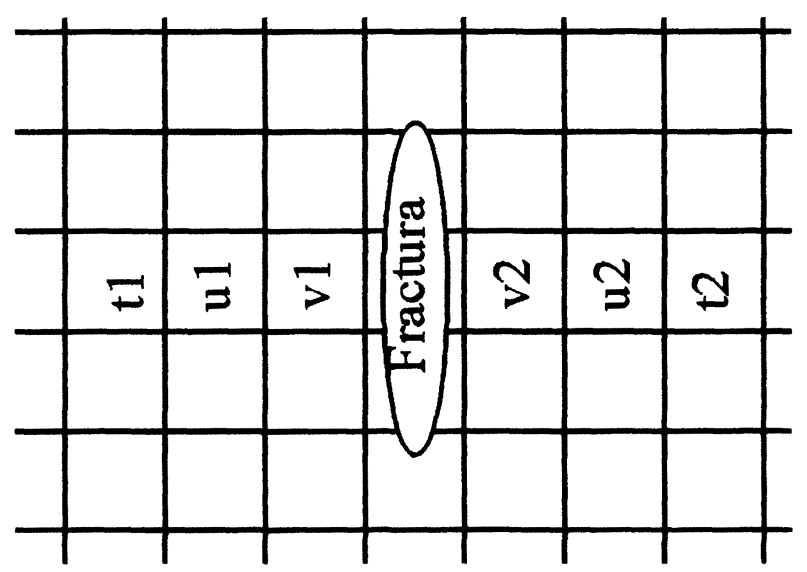

FIg.10.- Local de medição das extensões utilizando a fórmula de interpolação de Veermans.

FIG. 10.- The principle of measuring the strain using Veermans interpolation formula.

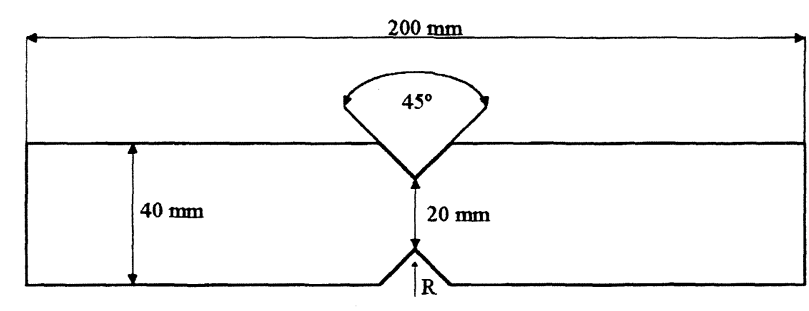

FIG. 11.- Provete utilizado nos ensaios de tracção, com diferentes valores de $\mathrm{R}$.

FIG. 11.- The shape of the tensile test blanks, with different radius.

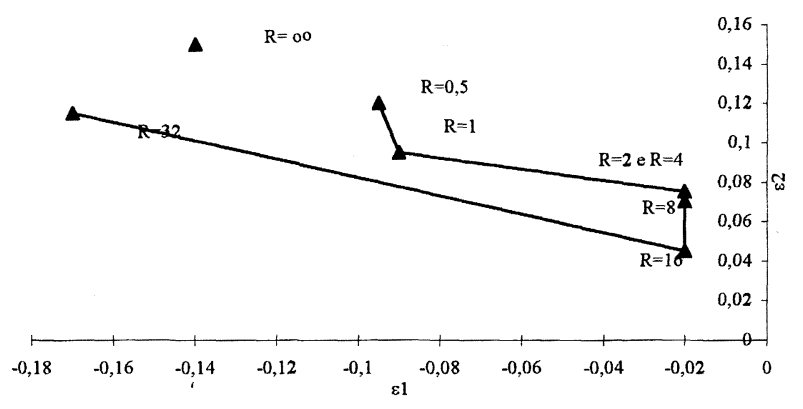

FIG. 12.- Relações entre as extensões obtidas nos ensaios de tracção.

FIG. 12.-Different tensile test blanks strain ratio.

elípticas, com três razões entre raios: 190/190, $150 / 190$ e 110/190. Estes quocientes são iguais aos quocientes entre as extensões. Neste teste a deformação foi imposta através da injecção de óleo na parte inferior da chapa, com caudal constante, pressionando a chapa contra a matriz, por forma a formar um hemisfério. As chapas foram deformadas até à instabilidade, tendo sido posteriormente medidas as extensões e estimadas as extensões na zona de fractura.

$\mathrm{Na}$ fig. 13 e 14 estão representadas as curvas limite de enformabilidade para as espessuras de 1,25 e $0,75 \mathrm{~mm}$, sendo cada ponto o valor médio de cinco medições. Ao lado de cada ponto está indicada o quociente entre as extensões. Para cada

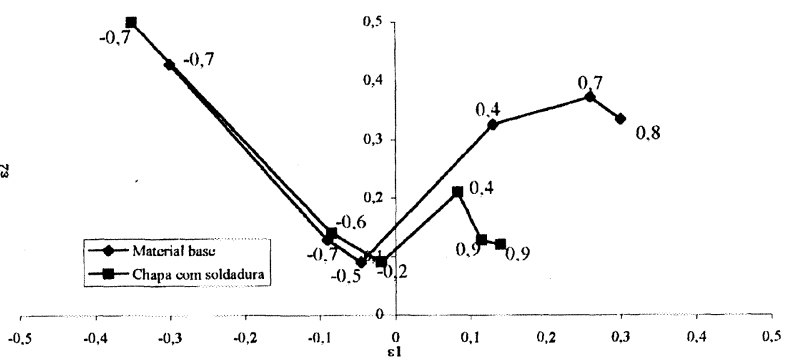

FIG. 13.- Curvas limite de enformabilidade para o aço St 1203 com $0,75 \mathrm{~mm}$ de espessura.

FIG. 13.-Forming limit curve 0,75 mm St 1203. 


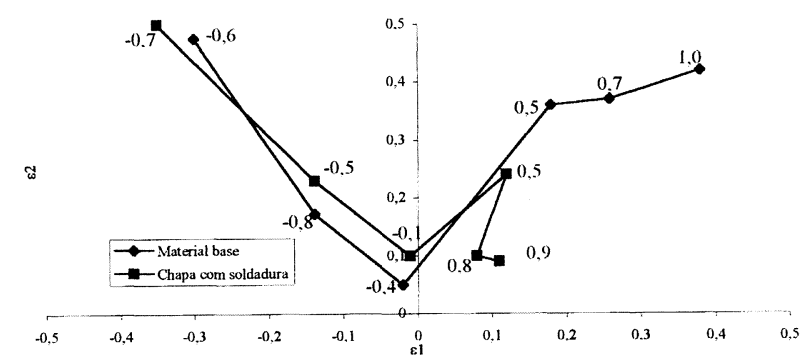

FIG. 14.- Curvas limite de enformabilidade para o aço St $1203 \mathrm{com} \mathrm{1,25} \mathrm{mm} \mathrm{de} \mathrm{espessura.}$

FIG. 14.- Forming limit curve 1,25 mm St 1203.

uma das espessuras está representada a variação da enformabilidade para o material base e para as chapas soldadas.

Da análise das curvas pode-se inferir que, do lado esquerdo das curvas o comportamento da chapa soldada é bastante idêntico ao do material base. Conclui-se portanto que não existe redução de enformabilidade das chapas soldadas, comparativamente com o material base, apresentando até valores superiores.

No lado direito das curvas verifica-se que o limite de enformabilidade é consideravelmente menor menor as chapas soldadas, existindo uma relação entre o quociente entre as extensões e a redução de enformabilidade. Quanto maior a quociente entre as extensões, menor o valor de extensão limite, ou seja, menor enformabilidade. Conclui-se portanto que a soldadura laser afecta a enformabilidade, a qual diminui se a deformação imposta originar quocientes entre extensões superiores a 0 .

\section{CONCLUSÕES}

Investigou-se neste artigo a formabilidade de chapas de aço St 1203 de 0,75 e 1,25 mm de espessuras, soldadas topo a topo por soldadura laser. Estudou-se a influência do desalinhamento vertical, para valores entre 0,1 e $0,5 \mathrm{~mm}$, tendo-se realizado testes de tracção e de embutição profunda. Deste testes concluiu-se que o valor crítico de desalinhamento está entre 0,3 e $0,4 \mathrm{~mm}$ para a espessura de $0,75 \mathrm{~mm}$.

Foi investigada a influência do decaimento do banho (com valores até $74 \mathrm{~mm}$ ) na enformabilidade, através da realização de ensaios de tracção. Os resultados demosntram que decaimentos de banho com valores até $74 \mathrm{~mm}$ não afectam a enformabilidade.

Utilizando o teste embutição (bulge test) e o ensaio de tracção foi possível construir as curvas limite de enformação para as espessuras de 0,75 e $1,25 \mathrm{~mm}$. Os resultados demonstram que para valores negativos de quociente entre extensões, a enformabilidade não é afectada; por outro lado para valores positivos deste quociente existe redução de enformabilidade da chapa soldada, em comparação ao material base.

\section{Agradecimento}

Esta investigação é parte do projecto "Laser Welding of Sub-Assemblies Before Forming", o qual é parcialmente financiado pela Comissão Europeia dentro do Programa CECA-ECSC.

\section{REFERENCIAS}

(1) ANDERSON S.G. "Review and forecast of laser markets: 1997-PartI" Laser Focus World vol. 33 n $^{\circ} 1$, ed. PennWell Publishing Co., p 72-92, Janeiro 1997.

(2) Belforte D.A. "Back on track for solid growth" Industrial Laser Review Vol.11 $\mathrm{N}^{\circ} 1$, ed. PennWell 'Publishing Co., p 25-29, Janeiro 1996.

(3) BELFORTE D.A. "Market growth exceeds expectactions" Industrial Laser Review Vol.12 $\mathrm{N}^{\circ}$ 1, ed. PennWell Publishing Co., p 19-24, Janeiro 1997.

(4) ANDERSON S.G. "Review and forecast of laser markets: 1996" Laser Focus World vol. 32 n $^{\circ} 1$, ed. PennWell Publishing Co., p 70-90, Janeiro 1996.

(5) ANDERSON S.G. "Review and forecast of laser markets: 1995" Laser Focus World vol. $31 \mathrm{n}^{\circ} 1$, ed. PennWell Publishing Co., p 74-95, Janeiro 1995

(6) IRving B. "Buillding tomorrow's automobiles", Welding Journal n 8 vol. 74, ed. American Welding Society p3934, Agosto 1995.

(7) Hoeven J.M.V. "Tailored-blanks: Actual and future innovation potencial in body construction", Proceeding of IBEC '96 - Materials \& Body Testing, ed. Automotive Technology Group, Inc., p 95-99, Detroit, Outubro 1996.

(8) Volvo "Laser/Robot cell for Volvo" Industrial Laser Review Vol.12 No 4, ed. PennWell Publishing Co., p 3, Abril 1997.

(9) IRVING B. "Welding tailored blanks is hot issue for automakers", Welding Journal $\mathrm{n}^{\circ} 8$ vol. 75, ed. American Welding Society, p 49-52, Agosto 1995.

(10) CoRrodi R. "European tailored-blank market: A challenge for competitors and suppliers for new applications", Proceeding of IBEC '96 - Materials \& Body Testing, ed. Automotive Technology Group, Inc., p 110-113, Detroit, Outubro 1996.

(11) BAYRON J. "An update on mash seam resistance welding" Welding Journal $\mathrm{n}^{\circ} 7$ vol. 73 , ed. American Welding Society p 35-39, Julho 1994.

(12) Malberg M., BAy N. "Instability curves by sheet metal forming", Technical University of Denmark, April 1994.

(13) Kristensen T., Olsen F. "Improvement laser welding using self-fixturing weld parts", Proceedings of Eurojoin 2, Florença, Itália, Maio 1994.

(14) Peças P., Henrique M., Miranda R.M., Quintino L. "Laser welding of low-thickness zinc-coated and uncoated carbon steel sheets", Optica and Quantum Electronics n²7, ed. Chapman \& Hall, p 1193-1201, 1995. 